\title{
Permalloy Multilayers to Reduce the Effects of Uniaxial Anisotropy
}

\author{
JOHAN W. WIEBERDINK AND KEES J. M. EIJKEL
}

\begin{abstract}
The anisotropic magnetoresistance effect of Permalloy $\left(\mathrm{Ni}_{0.82} \mathrm{Fe}_{0.18}\right)$ is used in a contactless angle detector [1]. The uniaxial magnetic anisotropy in a Permalloy thin film causes a difference between the magnetization and the magnetic field direction. This leads to a systematic error in the angle detector output. The effects of uniaxial magnetic anisotropy can be reduced by using Permalloy multilayers with different orientation of their anisotropy axes. A double layer with mutually perpendicular anisotropy axes is found to be sufficient for application in an angle detector. The Stoner-Wohlfarth single-domain model is used to describe the systematic error of an angle detector using multilayers.
\end{abstract}

\section{INTRODUCTION}

$\mathbf{I}_{\mathrm{b}}^{\mathrm{N}}$ N OUR research group a contactless angle detector based on the anisotropic magnetoresistance effect (AMR effect) in a Permalloy thin film has been proposed [1]. The AMR effect is used to detect the direction of magnetization $\bar{M}$ in the Permalloy film which is influenced by the magnetic field $\overline{\boldsymbol{H}}$ of a rotatable permanent magnet. The output signal of the angle detector yields the direction of magnetization (output angle $\theta$ ) while the object is to measure the angular position of the magnet (input angle $\varphi)$.

The uniaxial magnetic anisotropy of Permalloy causes a difference between the angles $\varphi$ and $\theta$ resulting in a systematic error $(\varphi-\theta)$ of the angle detector (see Fig. $1)$. This systematic error is determined by the strength and direction of both the uniaxial anisotropy (UA) and the magnetic field $\overline{\boldsymbol{H}}$ (Fig. 2). The UA is induced during the deposition of a Permalloy film and can be oriented with the aid of an external magnetic field during film growth. The orientation of the anisotropy axis or easy axis (EA) and the strength of the UA are characterized by the angle $\alpha$ (Fig. 1) and the anisotropy field strength $H_{k}$.

It is possible to calculate the systematic error if $\alpha$ and the ratio $H_{k} / H$ are known. In this way the angle detector output can be corrected. However, it is preferable to eliminate the effects of the UA in the Permalloy film itself. Annealing is one well-known means to reduce the UA [2], [3] and in our case a $1.5 \mathrm{~h} / 500^{\circ} \mathrm{C}$ annealing treatment proved to be sufficient to meet the requirements, viz. $(\varphi-\theta) \leq 0.1^{\circ}$ for $H=10 \mathrm{kA} / \mathrm{m}$. Another solution

Manuscript received December 1, 1988; revised February 2, 1989. Thi work was supported by the Netherlands Technology Foundation (STW)

The authors are with the University of Twente, EL-TDM, P.O. Box 217, 7500 AE Enschede, The Netherlands.

IEEE Log Number 8927698

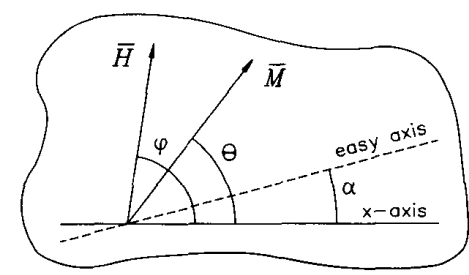

Fig. 1. A Permalloy film with uniaxial anisotropy.

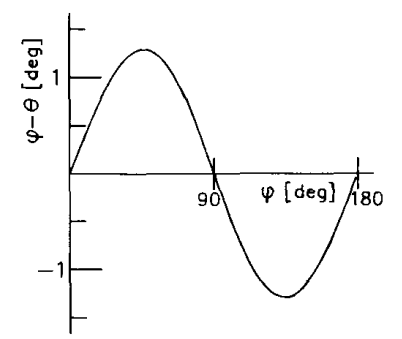

Fig. 2. The systematic error $(\varphi-\theta)$ of the angle detector as a function of $\varphi$.

exploits the inhomogeneity of the magnetic field of the permanent magnet to reduce the systematic error [3]. In this paper a new approach to reduce the effects of UA is proposed using multilayers of Permalloy with different orientation of their easy axes.

\section{Single Layer}

We start by describing the magnetic behavior of a single layer in order to describe the systematic error of an angle detector using multilayers. For a Permalloy film with UA in a homogeneous magnetic field the angle $(\varphi-\theta)$ follows the Stoner-Wohlfarth single-domain model [4]:

$$
\sin (\varphi-\theta)=\frac{H_{k}}{2 H} \sin 2(\theta-\alpha) .
$$

One cannot solve $\theta(\varphi)$ explicitely, but (1) can be rewritten as an iteration process (2). Now $\Theta(\varphi)$ can be calculated for all values of $\varphi$ starting with $\theta_{0}=\varphi$ and the exact solution can be approximated as accurately as necessary.

$$
\Theta_{i}=\varphi-\arctan \left(\frac{H_{k}}{2 H} \sin 2\left(\theta_{i-1}-\alpha\right)\right) .
$$


Alternatively, one can approximate $(\varphi-\theta)(\varphi)$ with the first two terms of a simple Fourier series derived from (1). This Fourier series (3) clearly shows how $(\varphi-\theta)$ depends on $\varphi$ and the ratio $H_{k} / H$ and is very appropriate for describing the systematic error of multilayers.

$$
\begin{aligned}
(\varphi-\theta)(\varphi) \approx & \frac{H_{k}}{2 H} \sin 2(\varphi-\alpha) \\
& -\left(\frac{H_{k}}{2 H}\right)^{2} \sin 4(\varphi-\alpha) .
\end{aligned}
$$

For a single-layer Permalloy film we have $H_{k} \approx 450$ $\mathrm{A} / \mathrm{m}$, so the maximum systematic error of the angle detector at $H=10 \mathrm{kA} / \mathrm{m}$ is $1.3^{\circ}$ (Fig. 2).

\section{Multilayers}

The effect of the UA in a Permalloy film on the performance of the angle detector can be reduced by using multilayers of Permalloy with different easy-axis orientation. This is obvious from the fact that a single layer with two mutually perpendicular easy axes with equal anisotropy constants $K_{u}$ is magnetically isotropic. The total anisotropy energy $E_{a}$ of such a film is independent of $\theta$ :

$$
E_{a}=K_{u} \sin ^{2} \theta+K_{u} \sin ^{2}\left(\theta+\frac{\pi}{2}\right)=K_{u}
$$

Metzdorf [5] managed to reorient a part of the anisotropy in the hard axis direction and realized isotropic Permalloy films. However, the magnetic properties of these films are not stable in a magnetic field for the desired operating temperatures of the angle detector. In order to approximate the ideal situation we realize multilayers with different EA orientation.

We used a computer simulation to calculate the systematic error of a double- and four-layer and determine its maximum value (Table I). Here, an ideal situation is considered: the $H_{k, i}$ and thickness $t_{i}$ of the layers ( $i=1,2$ or $1 \cdots 4$ ) are equal and the angle between the EA of subsequent layers is exactly $\pi / 2$ (double-layer) or $\pi / 4$ (four-layer). The influence of exchange and magnetostatic interaction between the layers is neglected.

A ferromagnetic exchange interaction between the layers would try to align their magnetization and would cause an additional decrease of the systematic error. An antiferromagnetic exchange interaction or a magnetostatic interaction would try to direct the magnetizations antiparallel and would increase the systematic error.

The computer simulation uses the iteration method (2) with the corresponding $\alpha_{i}$ to calculate the exact magnetization direction in each layer of a multilayer film. The average magnetization direction of a film, $\tilde{\theta}(\varphi)$, is defined as the average value of $\Theta_{i}$ over the number of layers. The averaging mechanism that occurs in the angle detector includes the nonlinear AMR effect, but for $H \gg H_{k}$ both averaging mechanisms provide approximately equal results. Table I shows the maximum values of $(\varphi-\tilde{\Theta})(\varphi)$ as a function of the number of layers (ver-
TABLE I

\begin{tabular}{|c|c|c|c|c|}
\hline \multirow{2}{*}{$\begin{array}{l}(\varphi-\tilde{\theta})_{\max } \\
\text { (in degrees) }\end{array}$} & \multicolumn{3}{|c|}{$H_{k} / H=$} & \multirow{2}{*}{$\begin{array}{c}\text { Function } \\
(\varphi-\tilde{\theta})(\varphi)\end{array}$} \\
\hline & 0.2 & 0.1 & 0.05 & \\
\hline Single-layer & 5.8 & 2.9 & 1.5 & $\sin (2 \varphi)$ \\
\hline Double-layer & 0.57 & 0.15 & 0.036 & $\sin (4 \varphi)$ \\
\hline Four-layer & 0.014 & 0.0089 & 0.000056 & $\sin (8 \varphi)$ \\
\hline
\end{tabular}

MAximum of $(\varphi-\tilde{\theta})(\varphi)$ OF MULTILAYers For Different Values of $H_{k} / H$

tical) and the value of $H_{k} / H$ (horizontal). In the last column the shape of $(\varphi-\tilde{\theta})$ as a function of $\varphi$ is given. From Table $\mathrm{I}$ it is clear that (5) approximates the systematic error of multilayers $\left(\alpha_{1}=0\right)$ :

$$
\begin{aligned}
& (\varphi-\tilde{\Theta})(\varphi) \approx C_{n}\left(\frac{H_{k}}{2 H}\right)^{n} \sin (2 n \varphi), \\
& \text { with } C_{1}=1, C_{2}=-1 \text {, and } C_{4}=2.5
\end{aligned}
$$

with $n$ being the number of layers. The same result can be obtained by using (3) for the successive Permalloy layers of a film. One simply has to add the expressions for $\left(\varphi-\theta_{i}\right)(\varphi)$ to obtain $(\varphi-\tilde{\theta})(\varphi)$. In case of a doublelayer the $\sin (2 \varphi)$ term is eliminated and in case of a fourlayer the $\sin (4 \varphi)$ term is also eliminated.

In a nonideal situation the above terms are not completely eliminated due to differences in the $H_{k, i}, t_{i}$, and $\alpha_{i}$ of the layers, caused by technological inaccuracies. In that case, $(\varphi-\tilde{\Theta})(\varphi)$ can be approximated with the first three terms of a Fourier series (6). The coefficients $c_{1}, c_{2}$, and $c_{4}$ denote the strength of the corresponding Fourier terms and depend on the ratio $H_{k} / H$ and the technological accuracy. The constants $\delta_{1}, \delta_{2}$, and $\delta_{4}$ represent the phase of the Fourier terms and depend on the field direction at $\varphi=0$ with respect to $\alpha_{1}$. The quality factors $q_{1}, q_{2}$, and $q_{4}$ defined in (6) are independent of $\boldsymbol{H}_{k} / \boldsymbol{H}$. They indicate the success of the technological realization. For an ideal double-layer we find $q_{1}=0$, while an ideal four-layer yields $q_{1}=q_{2}=0$.

$$
\begin{aligned}
(\varphi-\tilde{\Theta})(\varphi)= & c_{1} \sin \left(2 \varphi+\delta_{1}\right)-c_{2} \sin \left(4 \varphi+\delta_{2}\right) \\
& +c_{4} \sin \left(8 \varphi+\delta_{4}\right)+\cdots \\
\approx & q_{1} \frac{H_{k}}{2 H} \sin \left(2 \varphi+\delta_{1}\right) \\
& -q_{2}\left(\frac{H_{k}}{2 H}\right)^{2} \sin \left(4 \varphi+\delta_{2}\right) \\
& +q_{4}\left(\frac{H_{k}}{2 H}\right)^{4} \sin \left(8 \varphi+\delta_{4}\right)
\end{aligned}
$$

If $(\varphi-\tilde{\Theta})(\varphi)$ is determined for a multilayer, the anisotropy field strength of the Permalloy can be calculated from the Fourier coefficients in (6). This is, of course, an approximation of the actual anisotropy field strengths $H_{k, i}$ of the different layers. The value of the anisotropy field strength calculated this way will be called $\tilde{H}_{k}$. It is 
derived from the first term in (6) which is completely present

$$
\left.\begin{array}{rl}
\text { double-layer: } \tilde{H}_{k} & =2 H \sqrt{c_{2}} \\
\text { four-layer: } \tilde{H}_{k} & =2 H \sqrt[4]{\frac{c_{4}}{2.5} .}
\end{array}\right\}
$$

\section{TECHNOLOGY}

The Permalloy ( 82 at $\% \mathrm{Ni}$ and 18 at $\% \mathrm{Fe}$ ) films are RF sputtered on oxidized silicon wafers and a fixed bias field of $2 \mathrm{kA} / \mathrm{m}$ is used during film growth to direct the easy-axis orientation. In order to realize multilayers with different EA in our sputtering system it is necessary to break the vacuum and rotate the wafer by hand.

In our case, the bias field is too weak to induce a new EA in the next layer so the EA of the previous layer is continued [6]. We used an intermediate layer of chromium to interrupt the continuity of the Permalloy film growth. Chromium is very suitable because a thin layer of $3 \mathrm{~nm}$ is sufficient for magnetic separation [7] while the resistivity of $\mathrm{Cr}$ is relatively high. Therefore, the $\mathrm{Cr}$ layer hardly influences, that is, short-circuits, the AMR effect in the Permalloy layers (thickness 25 or $50 \mathrm{~nm}$ ) and does not disturb the AMR signal of the angle detector.

Application of a ferromagnetic intermediate layer would establish exchange interaction between the Permalloy layers and would further reduce $(\varphi-\tilde{\Theta})(\varphi)$. However, the actual advantage is negligible, because the maximum angle between the magnetization directions in neighboring films is small for the field strengths used in the angle detector. Consequently, the domain wall between subsequent Permalloy layers, having a width of a few nanometers, is restricted to the intermediate layer, so the exchange interaction has no influence on the magnetization orientation in the Permalloy layers. In order to obtain a symmetrical structure, extra $\mathrm{Cr}$ bottom and top layers are needed (Fig. 3). Otherwise, the values of $H_{k, i}$ and coercivity $H_{c, i}$ of separate Permalloy layers differ considerably.

In practice, three technological problems remain: The accuracy of the angle between the easy axes is determined by both the inhomogeneity of the bias field and the manual rotation of the wafer. Thickness variations within one layer up to 10 percent occur in our sputtering system due to the inhomogeneous sputter process, causing thickness differences between the layers of a multilayer film. This is a consequence of the small sputter target and the influence of the bias field on the plasma distribution.

\section{Characterization}

An Inductive Hysteresis Meter (IHM) is used to determine the $H_{c, i}, H_{k, i}$, and saturation magnetic moment $\mathfrak{M}_{s, i}$ of both layers $(i=1,2)$ of a double-layer. The layer thickness $t_{i}$ is assumed to be proportional to $\mathfrak{K}_{s, i}$. The $\mathfrak{M}-H$ curve of a double-layer is a superposition of the two $\mathfrak{M}-H$ curves of the separate layers. If the IHM measures
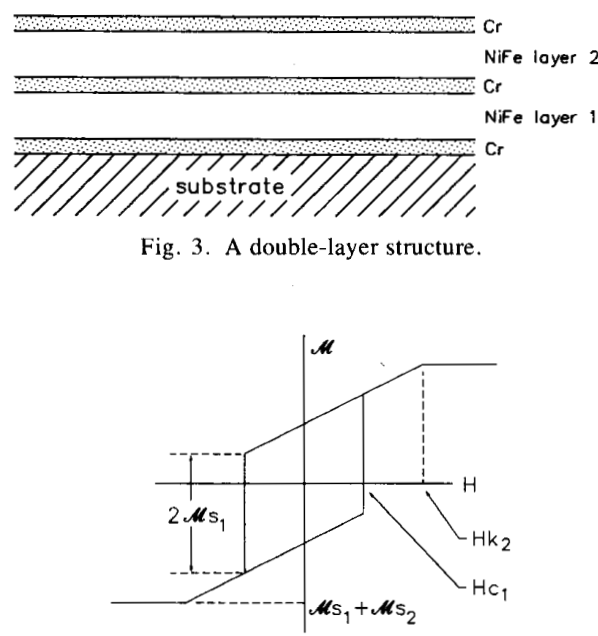

(a)

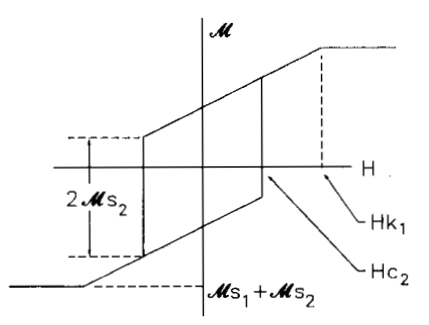

(b)

Fig. 4. $\mathfrak{M}-H$ curve EA1 + HA2 (a) and EA2 + HA1 (b) of a doublelayer.

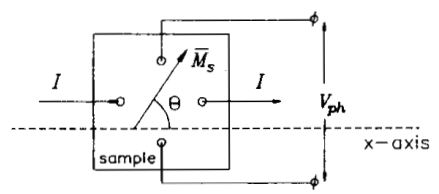

Fig. 5. A sample with point contacts in pseudo-Hall configuration.

the easy-axis curve of layer 1 (EA1), it also measures the hard-axis curve of layer 2 (HA2), because EA1 coincides with HA2 in a double-layer. Fig. 4(a) shows the $\mathfrak{N}-H$ curve EA1 + HA2 from which $H_{c, 1}, \mathfrak{N}_{s, 1}$, and $H_{k, 2}$ are determined. After rotating the film $90^{\circ}$, EA2 + HA1, with $H_{c, 2}, \mathfrak{N}_{s, 2}$, and $H_{k, 1}$, is measured (Fig. 4(b)). A fourlayer cannot be characterized with the IHM.

The Crowther method [8] is normally used to measure the angular dispersion in the easy-axis orientation. We used it to determine the exact angle between the different easy axes of a multilayer.

A third characterization method measures the Permalloy film in a pseudo-Hall configuration (Fig. 5). The film with voltage and current point contacts is rotated over $0^{\circ} \leq \varphi \leq 180^{\circ}$ in a magnetic field $H \leq 6 \mathrm{kA} / \mathrm{m}$ and the pseudo-Hall voltage $V_{\mathrm{ph}}(\varphi)$ is measured [9]. A computer program calculates $\Theta(\varphi)$ and determines the Fourier components of $(\varphi-\tilde{\theta})(\varphi)$. 


\section{Results AND Discussion}

We sputtered several double-layer films, with different $\mathrm{Cr}$ thickness $t_{\mathrm{Cr}}$, and one four-layer film. The sputtered wafers were broken into samples of $1 \times 1 \mathrm{~cm}^{2}$. The coercivity $H_{c}$ of a double-layer is reduced $\left(H_{c} \approx 25 \mathrm{~A} / \mathrm{m}\right.$ at $\left.t_{\mathrm{Cr}}=3 \mathrm{~nm}\right)$ and our results concerning $H_{c}$ as a function of $t_{\mathrm{Cr}}$ agree with Herd and Ahn [10]. The differences between the $H_{k, i}$ and $t_{i}(i=1,2)$ of a double-layer sample vary up to 10 percent. The maximum deviation of the easy-axis orientation with regard to the ideal orientation is found to be $7^{\circ}$. Each wafer contains some samples with a deviation of less than $1^{\circ}$. In general, the best samples are positioned at the center of a wafer and the relation between the technological success of a sample and $(\varphi-\tilde{\Theta})(\varphi)$ is always very obvious. We present the results of a successful double-layer and four-layer sample.

The systematic error $(\varphi-\tilde{\theta})(\varphi)$ of a double-layer (Fig. 6) is a $\sin (4 \varphi)$ function with an amplitude approximately proportional to $\left(H_{k} / 2 H\right)^{2}$. For higher values of the magnetic field strength (not shown in Fig. 6), the $\sin (2 \varphi)$ term with amplitude $q_{1} H_{k} / 2 H$ dominates in $(\varphi-\tilde{\theta})(\varphi)$. The factor $q_{1}$ indicates the suppression of the $\sin (2 \varphi)$ term and should be zero for an ideal doublelayer. The amplitude of $(\varphi-\tilde{\theta})(\varphi)$ is slightly higher than expected from the model. Therefore, the $\tilde{H}_{k}$ of a double-layer, calculated using (7), is increased in comparison with the $H_{k, i}$ of the separate layers. $\tilde{H}_{k}$ is found to depend on the magnetic field strength and increases up to 600 A $/ \mathrm{m}$ at $H=6 \mathrm{kA} / \mathrm{m}$. This is not predicted by the model.

The systematic error $(\varphi-\tilde{\Theta})(\varphi)$ of a four-layer (Fig. $7)$ is, in an ideal case, a $\sin (8 \varphi)$ function with an amplitude proportional to $\left(H_{k} / 2 H\right)^{4}$. The sin $(8 \varphi)$ term in the film of Fig. 7 is only recognizable for the lowest magnetic field strength $(500 \mathrm{~A} / \mathrm{m})$ because the $\sin (2 \varphi)$ term dominates for $H \geq 1 \mathrm{kA} / \mathrm{m}$. The influence of the $\sin (4 \varphi)$ term with amplitude $q_{2}\left(H_{k} / 2 H\right)^{2}$ is negligible because $H_{k} / H \ll 1$. Good four-layers $\left(q_{1}=q_{2}=0.01\right)$ can achieve $(\varphi-\tilde{\Theta})(\varphi) \leq 0.05^{\circ}$ for $H=5 \mathrm{kA} / \mathrm{m}$.

Again, the behavior of the film deviates from the model: The $\tilde{H}_{k}$ of the four-layer, calculated using (7), depends strongly on the magnetic field strength and varies from $350 \mathrm{~A} / \mathrm{m}$ at $H=500 \mathrm{~A} / \mathrm{m}$ to $1 \mathrm{kA} / \mathrm{m}$ at $H=6 \mathrm{kA} / \mathrm{m}$.

The unexpected dependence of $\tilde{H}_{k}$ on $H$ does not occur in single-layer films. Therefore, the observed deviation from the model is ascribed to the existence of magnetic coupling between the layers, which is neglected in the model. To explain the observed effects, this coupling should tend to increase the angle between the magnetization in two neighboring layers. Such a coupling can be either magnetostatic, viz. dipolar coupling via planar demagnetization, or of an exchange type. Computer simulations show that the demagnetizing field of our films is orders of magnitude too small to account for the observed behavior. Therefore, the existence of an antiferromagnetic exchange coupling between the Permalloy layers is regarded as a candidate for the observed deviations. Such an interaction between $\mathrm{Fe}$ thin films across a $\mathrm{Cr}$ intermediate layer has recently been reported by Grünberg [11].

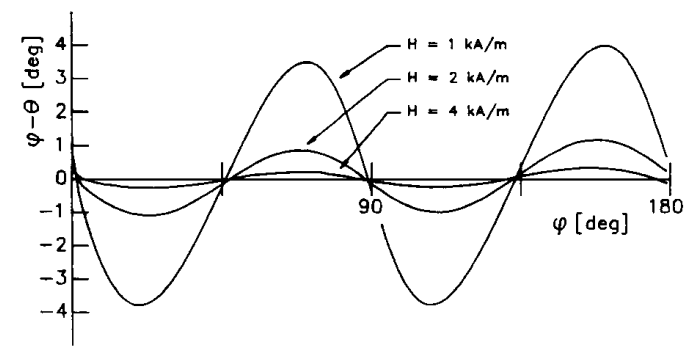

Fig. 6. Measured $(\varphi-\tilde{\theta})(\varphi)$ of a double-layer. $\left(t_{\mathrm{Cr}}=3 \mathrm{~nm}, t_{\mathrm{NiFe}}=\right.$ $50 \mathrm{~nm}, H_{k . i}=400 \mathrm{~A} / \mathrm{m}, q_{1} \approx 0.02$.)

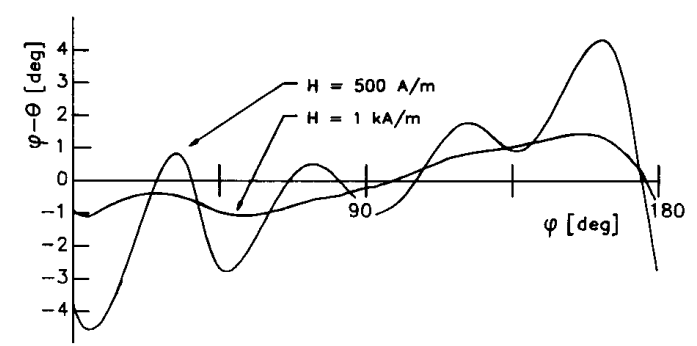

Fig. 7. Measured $(\varphi-\tilde{\theta})(\varphi)$ of a four-layer. $\left(t_{\mathrm{Cr}}=3 \mathrm{~nm}, t_{\mathrm{NiFe}}=25\right.$ $\mathrm{nm}, q_{1} \approx 0.1, q_{2} \approx 0.1$.)

His experiments concern epitaxially grown $\mathrm{Fe}$ and $\mathrm{Cr}$ layers, which seems to be of crucial importance for the observed pronounced antiferromagnetic coupling between the Fe layers. The question whether a weak antiferromagnetic exchange coupling between polycrystalline Permalloy layers across a thin polycrystalline $\mathrm{Cr}$ intermediate layer is the cause of the observed dependence of $\tilde{H}_{k}$ on $H$ is a very interesting one. Further experiments will be necessary to provide an insight into this problem.

\section{Conclusions}

The effect of the uniaxial anisotropy on the performance of the angle detector can be reduced by using multilayers of Permalloy with different easy-axis orientation. The anisotropy field strength $H_{k}$ and the thickness of each layer must be equal and the angle between their easy axes must be exactly $\pi / 2$ (double-layer) or $\pi / 4$ (four-layer). In this ideal situation, the systematic error $(\varphi-\tilde{\Theta})(\varphi)$ of the angle detector is in good approximation proportional to $\left(H_{k} / 2 H\right)^{n} \sin (2 n \varphi)$, where $n$ is the number of layers $(n=2,4)$.

In order to induce a new casy-axis orientation during deposition of a Permalloy layer on top of another Permalloy layer in a weak bias field, an intermediate layer is necessary to interrupt the continuity of the Permalloy, because otherwise the easy-axis orientation of the previous layer is continued. Chromium proved to be successful and has the advantage of not disturbing the AMR signal of the angle detector.

We realized double-layers that meet the requirements for application in an angle detector: $(\varphi-\tilde{\Theta})(\varphi)<0.1^{\circ}$ for $H=10 \mathrm{kA} / \mathrm{m}$. The model predicts an even better performance of four-layers but they require a more accu- 
rate technology. Our laboratory conditions are insufficient to realize good four-layers. The amplitude of $(\varphi-\tilde{\theta})(\varphi)$ of double- and four-layers is higher than predicted by the model. As a consequence, calculations based on the model yield an average anisotropy field strength $\tilde{H}_{k}$ of a multilayer that is higher than the $H_{k, i}$ of the separate layers. This is probably due to the neglect of magnetic coupling between the layers. Antiferromagnetic exchange coupling between the Permalloy and chromium layers is a possible candidate for such a coupling. Further experiments have to be performed to solve this question.

\section{ACKNOWLEDGMENT}

This work is part of the research on Permalloy sensors in the Transducers and Materials Science group of the Faculty of Electrical Engineering, University of Twente, under the supervision of prof. dr. Th. J. A. Popma and prof. dr. J. H. J. Fluitman. The authors would like to thank ir. P. de Haan and dr. R. M. de Ridder for helpful discussions.

\section{REFERENCES}

[1] K. J. Eijkel and J. H. J. Fluitman, "Angle detection based on the resistance anisotropy of permalloy," IEEE Trans. Magn., vol. MAG 22, pp. 955-957, 1986.

[2] J. W. Wieberdink, M. Sc. thesis, University of Twente, UT/EL/TDM $070.2386,1988$ (in Dutch).

[3] K. J. Eijkel and R. Rijk, "Contactless angle detection using permalloy," IEEE Trans. Magn., vol. 24, pp. 1761-1763, 1988.
[4] M. Prutton, Thin Ferromagnetic Films. London, UK: Butterworth, 1964 , p. 55.

[5] W. Metzdorf, "Die Beëinflussbarkeit der uniaxialen Anisotropie aufgedampfter Permalloyschichten durch Tempern im Magnetfeld," Zeitschrift Ang. Phys., vol. 18, pp. 534-540, 1965 (in German).

[6] K. J. Eijkel, "A thin film magnetoresistive angle detector," Ph.D. dissertation, University of Twente, 1988.

[7] P. Swiatek, F. Saurenbach, Y. Pang, P. Grünberg, and W. Zinn, "Exchange coupling of ferromagnetic films across nonmagnetic interlayers," J. Appl. Phys., vol. 61, pp. 3753-3755, 1987.

[8] T. S. Crowther, "Angular and magnitude dispersion of the anisotropy in magnetic films," J. Appl. Phys., vol. 18, pp. 580-586, 1963.

[9] K. J. Eijkel, "Measurement of the anisotropy in permalloy," IEEE Trans. Magn., vol. 24, pp. 1957-1959, 1988.

[10] S. R. Herd and K. Y. Ahn, "Magnetic domain structures in multilayered NiFe films," J. Appl. Phys., vol. 50, pp. 2384-2386, 1979

[11] P. Grünberg in R. F. C. Fallow et al., Thin Film Growth Techniques for Low Dimensional Structures (NATO ASI Series, Series B Physics Vol. 163). New York, NY: Plenum, 1987, pp. 487-505.

Johan W. Wieberdink was bom in Apeldoorn, The Netherlands, in 1963 He received the $M$.Sc. degree in electrical engineering from the University of Twente, Enschede, The Netherlands, in 1988.

Kees J. M. Eijkel was born in Heiloo, The Netherlands, in 1959. He received the M.Sc. degree in mathematics from the University of Amsterdam, Amsterdam, The Netherlands, in 1983 and the Ph.D. degree from the University of Twente, Enschede, The Netherlands, in 1988. 